\title{
Mitral Regurgitation on Native Valve with Ostium Secundum Atrial Septal Defect (ASD) Realizingtiger Stripes Sign that Disappear after Mechanical Valve Replacement Case Report with Literature Review
}

\author{
${ }^{1}$ EL ABASSE ZEINE MD \\ ${ }^{2}$ BAH ALI RIDA MD \\ ${ }^{3}$ Taouakalna amira MD \\ ${ }^{4}$ HABBAL RACHIDA, MD ; PHD. \\ ${ }^{5}$ ETTAOUMI YOUSSEF MD ; PHD. \\ 1,2,4 CARDIOLOGIST HOSPITAL CENTER UNIVERSITY IBN ROCHD CASABLANCA MOROCCO. \\ 3,5 cardiovascular surgeon HOSPITAL CENTER UNIVERSITY IBN ROCHD CASABLANCA MOROCCO.
}

\section{Abstract:-}

\section{> Background \\ Tiger stripes sign is the high amplitude band-like signal noted on Doppler spectral recordings of valvular regurgitation. This is noted most often in patients with porcine mitral prosthetic valves and may be indicative of flail prosthetic leaflet. We herein describe a patient with tiger stripes signin a severe mitral regurgitation on native valve associated with Ostium secundum atrial septal Defect (ASD).}

\section{Case summary}

A 41-year-old woman hospitalized for progressively worsening exertional dyspnea NYHA (New York Heart Association) class III associated with chest pain evolving for the past 3 years. The patient's medical history includes Grave's disease. Clinical examination revealeda grade 5/6 blowing holosystolic murmur at the apex and a grade $4 / 6$ blowing murmur at the tricuspid area.ECG showed trial Fibrillation. Echocardiography showed the presence of severe mitral regurgitation Figure 1: (A), severe tricuspid regurgitation and secundum atrial septal defect with $10 \mathrm{~mm}$ diameter. Doppler showed the appearance of tiger stripes sign in continuous-wave doppler $(\mathrm{CW})$ and in pulsed-wave doppler $(\mathrm{PW})$ on the mitral valve. Figure 1: $(B, C)$.

\begin{abstract}
$>$ Discussion
Tiger stripes sign is the high amplitude band-like signal noted on Doppler spectral recordings (pulsed and continuous both) of valvular regurgitation. Other conditions associated with tiger stripes sign are any intracardiac oscillating mass and par prosthetic leak. Though the mechanism of its formation is still not clear. In our case, tiger strips sign was present in a patient with severe mitral regurgitation on native valve associated with ostium secundum atrial septal defect. The purpose of this report is to help us better understanding the path physiological mechanism of this sign.
\end{abstract}

\section{Conclusion:}

The presence of regurgitation with divergent jet associated with ASD can favor the appearance of the tiger stripes sign.

\section{INTRODUCTION}

Tiger stripes sign (also known as Zebra stripes sign) is the high amplitude band-like signal noted on Doppler spectral recordings of valvular regurgitation. This is noted most often in patients with porcine mitral prosthetic valves and may be indicative of flail prosthetic leaflet.1,2To the best of our knowledge, there is only few reports in the literature regarding tiger stripes sign. We herein describe another patient who presents with a particularity that has never been described before; the appearance of tiger stripes sign in a severe mitral regurgitation on native valve associated with Ostium secundum atrial septal Defect (ASD). 
II. TIMELINE

\begin{tabular}{|l|l|}
\hline 3 years prior to admission & Progressive dyspnea associated with palpitation and intermittent headache. \\
\hline Admission : Cardiac convention care unit & $\begin{array}{l}\text { Evaluation during admission: } \\
\text { Electrocardiogram: Atrial Fibrillation with heart rate of } 70 \mathrm{bpm} . \\
\text { Cardiac monitoring: Atrial Fibrillation with no arrhythmias } \\
\text { Echocardiography: Good ventricular function, left ventricular ejection fraction } \\
\text { (LVEF) 57\%. Severe Mitral Regurgitation. Right cavities dilated, with good } \\
\text { longitudinal systolic function. Ostiumsecundum ASD was 10 mm diameter; severe } \\
\text { tricuspid insufficiency with Pulmonary arterial systolic pressure was 55+15 mmHg. } \\
\text { We noted the appearance of tiger stripes sign in continuous-wave doppler (CW) and } \\
\text { in pulsed-wave dopller (PW) on the Mitral valve Figure 1: (B, C). }\end{array}$ \\
\hline Surgical treatement & $\begin{array}{l}\text { The patient underwent: } \\
\text { Mitral valve replacement with mechanical prosthesis because the valve was not } \\
\text { repairable, tricuspid annuloplasty using the De Vega technique, and ASD closure. } \\
\text { Figure 1:(D). }\end{array}$ \\
\hline Following discharge & $\begin{array}{l}\text { Good clinical course, dyspnea was down to NYHA class II, with good prosthesis } \\
\text { mitral valve functioning. } \\
\text { TTE control: we have reported the disappearance of tiger stripes sign. Figure } \\
\text { 1:(E,F). }\end{array}$ \\
\hline
\end{tabular}

\section{CASE PRESENTATION}

A 41-year-old woman hospitalized in our cardio department for progressively worsening exertional dyspnea NYHA class III associated with chest pain evolving for the past 3 years (It should be noted that the patient was been diagnosed with valve disease 3 years ago and she did not show-up for follow-up visits since then). The patient's medical history includes Grave's disease that was been treated by radioactive iodine therapy for 4 weeks and carbimazole intake. Clinical examination revealed a normal blood pressure $120 / 79 \mathrm{mmHg}$ with pulse rate of $71 \mathrm{bpm}$, a grade 5/6 blowing holosystolic murmur at the apex along with a grade $4 / 6$ blowing murmur at the tricuspid area. The biological test showed: hypochromic microcytic anemia (Haemoglobin $9.2 \mathrm{~g} / \mathrm{dl}$ ), normal leucoytes and low platelets counts (4170 and 129000 cells/uL, respectively); TSH inferior than $0.005 \mathrm{uUI} / \mathrm{mL}$ with normal T3 and T4 (5.7 and $15.7 \mathrm{pg} / \mathrm{ml}$, respectively).

Electrocardiogram: Atrial Fibrillation with heart rate of $70 \mathrm{bpm}$.

ECHOCARDIOGRAPHY: left ventricledilatedwith good ventricular function, left ventricular ejection fraction (LVEF) $57 \%$.Severe mitral very eccentric Regurgitation (vena contracta $0.9 \mathrm{~cm}$, effective regurgitant orifice (ERO) $0.8 \mathrm{~cm}^{2}$, regurgitant volume (Rvol) $100 \mathrm{ml}, \mathrm{LV}$ dilatation), organo-functional annular dilationup to $50 \mathrm{~mm}$; with restriction of the small and dislocation of the large valve. Figure 1: (A).

Right cavities Dilated, with good longitudinal systolic function andfractional area change (FAC) $36 \%$. Ostium secundum ASD was $10 \mathrm{~mm}$ diameters. Severe tricuspid regurgitation (proximal-isovelocity-surface-area (PISA)
$9 \mathrm{~mm}$, ERO $0.5 \mathrm{~cm}^{2}$ ) functional by annular dilation up to $45 \mathrm{~mm}$ with pulmonary arterial systolic pressurewas $55+15$ $\mathrm{mmHg}$.We noted the appearance of tiger stripes sign in continuous-wave doppler $(\mathrm{CW})$ and in pulsed-wave doppler (PW)on the mitral valve. Figure 1: $(\mathrm{B}, \mathrm{C})$.

Transeosophageal echocardiography (TEE) was highly recommended to complete and evaluate more the mechanism of the valves disease and ASD, but the patient refused the test.

The patient underwent a mitral valve replacement with mechanical valve, tricuspid annuloplasty using the De Vega technique and ASD closure. Figure 1: (D).

Postoperative period was uneventful, with good clinical outcomes. The patient's dyspnea was down to NYHA class II with good prosthesis mitral valve functioning and most importantly, we have noted the disappearance of tiger stripes sign. Figure 1:(E,F).

\section{DISCUSSION}

Tiger stripes sign is the high amplitude band-like signals seen in Doppler spectral recordings (pulsed and continuousboth) of valvular regurgitation. This was usually considered to indicate the presence of a flail porcine prosthetic mitral leaflet. 1,2 However; it can be associated with any other intracardiac oscillatory mass or vibratory structure, 3,4 or even with native valve regurgitation, as was reported by Goyal (Goyal et al. 2018) 5 and Rakesh (Rakesh et al. 2015).6

The tiger stripes sign is not specific to a certain valvular. A case reported by Muthiah (Muthiah 2018) showed the appearance of tiger stripes at the tricuspid valve 
due to the oscillating mass across the valve. 7 as well as Sahu's case (Sahu et al 2016) showed the tiger stripes within the tricuspid regurgitation arising probably due to abnormal septal tricuspid leaflet oscillatory motion with the jet of TR. 8-9 Another case reported by Muthiah (Muthiah 2016) showed the appearance of tiger stripes on spectral Doppler of mitral valve due to oscillating flail segments in the regurgitant stream. 9 and more particulary, the Davogustto's case (Davogustto et al. 2015) showed the presence of tiger stripes sign in a patient with Lambl's excrescences in Aortic valve. 10

The definite mechanism leading to the formation of tiger stripes is still not clear. It was believed to be caused by fluttering of the disrupted cusp leading to shedding of vortices in both retrograde and orthograde direction. 2 However, it is considered that any intracardiac oscillating structure vibrating with a single high frequency (not chaotic) with several harmonic overtones can give rise to tiger stripes sign. With the first band (lowest frequency on the Doppler recording) representing its fundamental frequency. 11,12 Also, the observed Doppler fundamental frequency (and amplitude) appears to be proportional to regurgitant flow rate across the valve.11 The number of striations in the signal is likely to be related to the frequency of oscillation of the cusp and the presence or absence of mottling to the pattern of shedding of the vortices, 2 also it can be related to the severity of the regurgitation. It is assumed that the streak numbers depend on the direction of blood flow, especially in the case of eccentric regurgitation. These tiger stripe frequencies may also occur in the absence of blood flow turbulence as seen in native valve. 3

In our case, the tiger stripes sign was been found on transthoracic echocardiography in severe mitral regurgitation on native valve associated with ostium secundum ASD. Which a transesophageal echocardiography was highly recommended to complete and evaluate more the mechanism of the mitral regurgitation and ASD, unfortunately the patient refused the procedure.

It is known that different etiological diagnosis of tiger stripes are valve regurgitation, flail prosthetic valve leaflet, intracardiac oscillatory mass and possibly Lambl's excrescences, as previously reported. We can include the association of eccentric mitral regurgitation and ASD. As we noticed in our case, the presence of regurgitation with divergent jet associated with ASD has favor the appearance of the tiger stripes sign.

We share another particularity in our case, is that the patient underwent surgery, unlike the prior cases reports. There was an indication for surgery, because the patient were symptomatic and her LVEF < 60\%; LVESD> 45mm; the presence of atrial fibrillation and a systolic pulmonary pressure $>50 \mathrm{mmHg}$ with severe tricuspid regurgitation by functional annular dilation $>40 \mathrm{~mm}$. As an outcome, we noted the disappearance of tiger strips sign after mitral valve replacement by mechanical valve and ASD closure.

\section{Case study Limitations}

Lack of prior research studies on this topic, as there were only few cases reported.

\section{CONCLUSION}

The tiger stripe sign is considered as a rare and an interesting echocardiography phenomena. In the presence of tiger stripes sign, the physician must above all eliminate prosthesis dysfunction, or an eccentric valve regurgitation associated or not with ASD. On the other hand, our case showed the disappearance of this sign after mechanical valve replacement and ASD closure, which is a particularity that has never been reported before in literature.

\section{DECLARATIONS}

- Ethics approval and consent to participate : Not applicable.

- Consent for publication : The authors certify that they have obtained

- Appropriate patient consent form. In the form the patient has consent for his images and other clinical information to be reported in the journal. The patient understand that their names and initials will not be published.

- Availability of data and material : Not applicable.

- Competing interests : The authors declare that they have no competing interests

- Funding: Not applicable.

- Authors' contributions: All authors read and approved the final manuscript.

\section{REFERENCES}

[1]. Feigenbaum $H$, Armstrong WF, Ryan T. Feigenbaum's Echocardiography. 7th ed. Philadelphia: Lippincott Williams \& Wilkins; 2010:318-319

[2]. Chambers JB, Monaghan MJ, Jackson G, et al. Doppler echocardiographic appearance of cusp tears in tissue valve prostheses. J Am Coll Cardiol. 1987; 10:462.

[3]. Pennestri F, Boccardi L, Minardi G. Doppler study of precordial musical murmurs. Am J Cardiol. 1989;63:1390-1394.

[4]. Jacovella G, Narducci C, Pino PG, Salati A. Echocardiographic features of porcine valve dysfunction. Am J Cardiol. 1980;46:526.

[5]. Goyal KK, Mukund DE, Sajeev CG. Tiger stripes sign. $\mathrm{J}$ Indian Acad Echocardiogr Cardiovasc Imaging. 2018;2:139-40

[6]. Rakesh J and Krishnan MN. Tiger stripes sign. Journal of Indian college of cardiology. 2015;5:138-40

[7]. Muthiah, R. (2018) Isolated Right-Sided Infective Endocarditis-An Infected Right Ventricular Myxoma Masquerading as "Tricuspid Vegetation"-A Case Report. Case Reports in Clinical Medicine, 7, 182-209

[8]. Sahu AK, Kapoor A. Heart Asia 2016;8:45. doi:10.1136/heartasia-2016-010841 
[9]. Kerut EK. Tiger stripes. Echocardiography 2007;24:558-9

[10]. Muthiah, R. (2016) Rheumatic Mitral Valvulitis with a "Giant Vegetation"-A Case Report. Case Reports in Clinical Medicine, 5, 363-410. http://dx.doi.org/10.4236/crcm.2016.510056

[11]. Davogustto G, Fernando RR, Loghin C. Lambl's excrescence, migrainous headaches, and "tiger stripes": Puzzling findings in one patient. Tex Heart Inst J 2015;42:70-2
[12]. Sabbah HN, Magilligan DJ Jr., Lakier JB, Stein PD. Hemodynamic determinants of the frequency and amplitude of a musical murmur produced by a regurgitant mitral bioprosthetic valve. Am J Cardiol 1982;50:53-8.

[13]. McKusick VA, Murray GE, Peeler RG, et al: Musical cardiovascular murmurs. Bull Johns Hopkins Hosp 1955;97:136-176.

Figure 1:
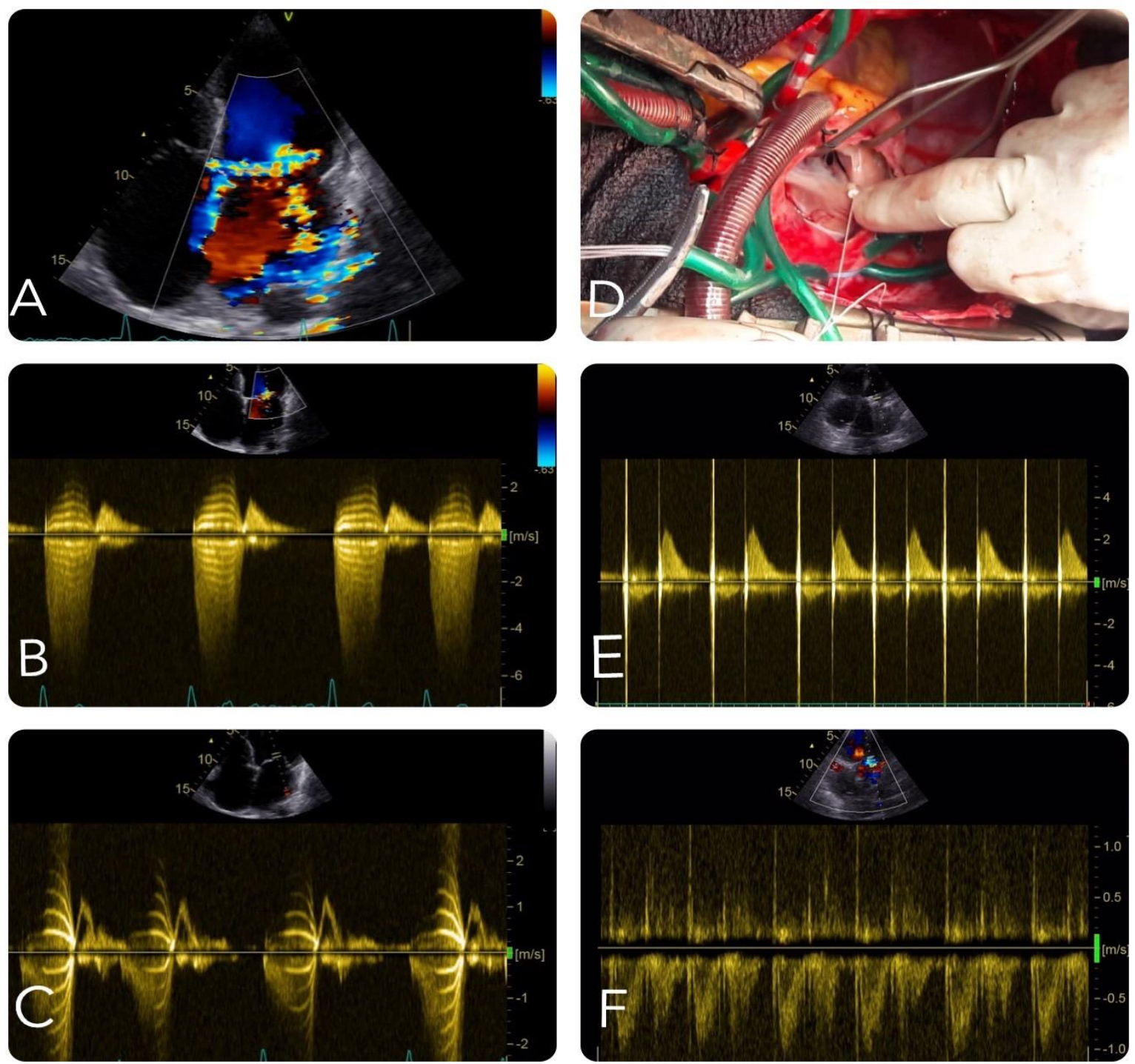

-A:Severe mitral very eccentric Regurgitation.

-B: Tiger stripes sign in continuous-wave doppler (CW).

-C: Tiger stripes signin pulsed-wave doppler (PW).

-D: Mitral valve replacement by mechanical valve and ASD closure.

-E: The disappearance of tiger stripes signin continuous-wave doppler (CW)Postoperative.

-F: The disappearance of tiger stripes signin pulsed-wave doppler (PW)Postoperative. 